\title{
Acceptability of a Cognitive Behavior Therapy Intervention to Implantable Cardioverter Defibrillator Recipients
}

\author{
Jane Irvine, $\mathrm{PhD}$ \\ Jill Stanley, PhD \\ Lephuong Ong, PhD \\ Robert Cribbie, $\mathrm{PhD}$ \\ Paul Ritvo, PhD \\ Joel Katz, PhD \\ York University \\ Paul Dorian, MD \\ Suzan O'Donnell, MSc \\ St. Michael's Hospital \\ Louise Harris, MD \\ Doug Cameron, MD \\ Ann Hill, RN
}

Toronto General Hospital, University Health Network

\section{David Newman, MD}

Sunnybrook Health Sciences Centre

\author{
Sabine N. Johnson, PhD \\ Ana Bilanovic, $\mathrm{PhD}$ \\ York University
}

Samuel F. Sears, Jr., PhD

East Carolina University

\begin{abstract}
We aimed to assess cardiac patients, acceptance of cognitive behavior therapy (CBT); determine if gender was associated with treatment engagement (session attendance and utilization of intervention strategies); and relate engagement to outcome. Of 193 patients receiving an implantable cardioverter defibrillator (ICD) who agreed to participate in a randomized controlled trial, 96 were randomized to CBT. Measures of treatment acceptance indicated that most participants rated counseling as "very to extremely helpful." Gender was associated with only one treatment engagement index. Symptoms of depression and post-traumatic stress improved from baseline to 6- and 12-month follow-up. Number of counseling session
\end{abstract}


attendance was not associated with outcome. Reported utilization of two of the six CBT strategies (modifying faulty thinking, correcting cognitive distortions) was associated with a better treatment outcome. In conclusion, a CBT intervention was well received by ICD patients. There was some indication that treatment engagement related to better treatment outcomes.

Keywords: treatment acceptance; cognitive behavior therapy; implantable cardioverter defibrillator

$\Theta^{t}$ tudies indicating associations between psychological disorders and increased risk of cardiac mortality (Frasure-Smith \& Lesperance, 2008; Irvine et al., 1999; Ladwig et al., 2008) have prompted intervention trials aimed at treating psychological disorders in this population (Linden, Stossel, \& Maurice, 1996). A challenge to intervening with medical populations however, is that these patients are not necessarily seeking or interested in receiving psychological interventions (Coyne, Lepore, \& Palmer, 2006). Moreover, investigators have cautioned that psychological interventions may not be uniformly benign (Coyne \& Lepore, 2006). Concerns regarding cancer patients' receptivity to psychological interventions have led to a debate about their acceptability, efficacy, and safety (Stefanek, Jacobsen, \& Christensen, 2006). Similar considerations apply to cardiac patients. Suggestions that specific cardiac patient subgroups (e.g., females, patients with higher levels of defensiveness) are especially adversely affected by psychological interventions (Frasure-Smith et al., 1997; Frasure-Smith et al., 2002; Sears, Todaro, Lewis, Sotile, \& Conti, 1999) support the need to examine psychological treatment acceptance, engagement, and outcome in this population.

\section{STUDY Aims}

The aims of this study were to assess participants' acceptance of cognitive behavior therapy (CBT) for facilitating adjustment to an implantable cardioverter defibrillator (ICD); determine if gender is related to treatment engagement; and assess the association between treatment engagement and psychological outcome. The current study was conceived after completing our randomized controlled trial (RCT) of CBT for patients receiving an ICD (Irvine, Firestone, Ong, Cribbie, Johnson, et al., 2009). Thus, the therapy engagement variables were limited to those available. The RCT compared psychological outcomes between patients randomized to CBT or usual cardiac care (UCC).

\section{INTERVENING WITH ICD RECIPIENTS}

Arguably, patients receiving an ICD are in particular need of support due to the stress of their life-threatening condition and the life-saving treatment. The ICD monitors malignant ventricular arrhythmias, treating them with anti-tachycardia pacing (ATP) or with high-energy shocks. Not only have elevated depressive (Irvine et al., 1999) and post-traumatic stress disorder (PTSD) symptoms (Ladwig et al., 2008) been associated with increased mortality in this population but the ICD itself is associated with increased psychological problems (Dunbar et al., 1999; Irvine, Dorian, et al., 2002).

There are now numerous studies of psychological interventions to aid adjustment to the ICD. A recent review of these studies found that more structured interventions, such as CBT, were more successful at reducing anxiety and depression than non-specific support interventions (Pedersen, van den Broek, \& Sears, 2007). However, most studies report low participation rates, ranging from $26 \%$ to $47 \%$, suggesting engagement challenges (Pedersen et al., 2007). 


\section{TREATMENT ENGAGEMENT}

Studies of treatment engagement in mental health populations (Carroll et al., 2008; Meyer et al., 2002) do not necessarily generalize to medical health populations. A notable difference between these two populations is that the former is seeking treatment for emotional problems, whereas the latter is not. Thus, medical patients' motivations for psychological interventions are likely at earlier stages of readiness for engagement (Prochaska et al., 1994) and may be more complicated by factors like treatment expectancy (Greenberg, Constantino, \& Bruce, 2006; Meyer et al., 2002), perceptions of control (Leung \& Heimberg, 1996), ambivalence about change (Westra, 2004), perceived responsibility for change (Delsignore, Carraro, Mathier, Znoj, \& Schnyder, 2008), and therapeutic alliance (Gaston, Marmar, Thompson, \& Gallagher, 1988).

\section{GENDER AND TREATMENT ENGagemENT}

Women have been found to experience more psychological problems following cardiac events (Pilote et al., 2007). Unfortunately, they may be less likely to respond to psychological interventions (Berkman et al., 2003; Frasure-Smith et al., 1997), and be less likely to participate in cardiac rehabilitation programs (Grace et al., 2002). Moreover, reviews of psychosocial outcomes in ICD patients indicate poorer outcomes in women (Sears, Vazquez, Matchett, \& Pitzalis, 2008). Similarly, women in our RCT of CBT scored significantly worse than men at baseline on psychological distress symptoms (Irvine, Firestone, Ong, Cribbie, Dorian, et al., 2009). The results of the RCT have been reported elsewhere (Irvine, Firestone, Ong, Cribbie, Johnson, et al., 2009) and indicated significant CBT treatment effects on depression and PTSD symptoms at 12-month follow-up (Irvine, Firestone, Ong, Cribbie, Johnson, et al., 2009). Surprisingly though, the CBT treatment effect on depressive symptoms was limited to women in the trial (Irvine, Firestone, Ong, Cribbie, Johnson, et al., 2009).

\section{TreatMent ACCEPTANCE}

Researchers have recommended that studies go beyond indexing engagement via participation and retention rates to now include measures of patient acceptance and opinions about the interventions provided (Andrykowski \& Manne, 2006). To our knowledge, none of the psychological trials with cardiac patients assessed patient's opinion of therapy elements. However, there are examples of treatment acceptance indices such as ratings of satisfaction, importance, and adequacy from studies in people with other health problems (Carroll et al., 2008; Cupertino et al., 2007). For the purposes of our study, we assessed engagement by asking participants to evaluate the helpfulness and importance of specific intervention elements.

\section{Measures of Treatment Engagement}

Treatment engagement has been assessed by adherence to homework (Carroll et al., 2008; Cowan et al., 2008; Fiorentine, Anglin, Gil-Rivas, \& Taylor, 1999) and session attendance (Cupertino et al., 2007; Fiorentine et al., 1999). We did not have a measure of homework adherence per se. Rather, participants' reports of using different CBT interventions were used as an index of engagement along with session attendance rates.

Higher treatment expectancies are thought to lead to greater engagement, which in turn relate to better outcomes (Carroll et al., 2008; Meyer et al., 2002; Westra, Dozois, \& Marcus, 2007). In this study, we tested the association between participants' reports of utilizing different intervention strategies and session attendance rates and outcome. 


\section{MethodS}

\section{Participants}

Patients from St. Michael's Hospital (SMH) and University Health Network (UHN) in Toronto, Canada, who received their first ICD for either secondary or primary prevention of sudden cardiac death (SCD) were recruited between October 2003 and August 2006. Exclusion criteria included inability to read or understand English; dementia, cognitive impairment, or psychosis documented in the medical chart; and ICD implant for primary prevention of SCD due to coronary heart disease and ischemic cardiomyopathy and congestive heart failure.

\section{Procedure}

This study was reviewed and approved by the Human Participants Review Committees of York University, the UHN, and SMH. Eligible patients were identified and recruited by the study nurses who also obtained the participant's written consent. The nurses were responsible for completing the baseline and follow-up clinical case report forms, and for sending the participant's consent form, clinical data, and questionnaire to the coordinating centre at UHN.

Participants completed the baseline battery of questionnaires prior to or shortly after hospital discharge. Every effort was made to keep the nurses and clinical staff blind to the group to which the participant had been randomized. Participants were randomized to receive either CBT or UCC within four blocking variables: hospital site (SMH or UHN), age ( $<70$ years vs. $\geq 70$ years), gender, and primary arrhythmia type (ventricular fibrillation [VF] or cardiac arrest vs. other). The CBT intervention participants were further randomized to one of the four counselors. Intervention materials were shipped to participants within a week of receiving their completed baseline questionnaires.

\section{Cognitive-Behavior Therapy Intervention}

The CBT intervention was designed to address patients' concerns about living with an ICD and to teach anxiety management skills. It included a therapist manual, eight counseling sessions, a psycho-educational booklet for the participants (Irvine, Ritvo, et al., 2002), and a compact disc (CD)-ROM with mindfulness-based and progressive muscle relaxation exercises. Participants were offered ad hoc counseling sessions if they experienced an ICD shock.

The original design included one face-to-face session scheduled to coincide with the participants' first ICD clinical follow-up visit, two group sessions scheduled at 10 and 14 weeks post implant, and five telephone sessions scheduled at 4, 8, 12, 16, and 20 weeks post implant. The telephone sessions were planned in order to overcome logistical barriers to participation due to geography (patients can live considerable distances from the ICD treatment hospital) and driving restrictions (patients are restricted from driving for at least 6 months post implant). After efforts to schedule group sessions proved very difficult, they were replaced with two telephone sessions. If logistically impossible to schedule, the face-to-face session was also replaced with a telephone session.

The focus of counseling was guided by the content of the psycho-educational booklet with the sequence of topics structured to be sensitive to the course of recovery and adaptation to an ICD. The implementation of counseling was client-centered. Thus, while a particular session began with reviewing the participant's progress from the previous session and the introduction of the next topic from the booklet, the session focus was ultimately determined by the participant's interest. A summary of the chapter themes from the booklet is presented in Table 1.

The anxiety-management interventions were based upon the cognitive theories of anxiety proposed by Beck (1976) and Salkovkis and Clark (Salkovskis, Clark, \& Gelder, 1996). In addition, 
Table 1. Chapter Outline of ICD Patient's Psycho-Educational Booklet

Chapter Title

"Answers to Your Questions"

"Energy: The Fuel in Your Tank"

"Finding the Support You Need"

"Feeling Good-Reducing Stress and Anxiety"

"Love and Intimacy"

"Last Words"
List of Topics

Information preferences

ICD recovery issues (e.g., bathing, arm movement)

How the ICD works

Daily living with an ICD

Patient accounts of how an ICD shock feels

Your shock action plan

Emotion \& energy flow (e.g., changing negative thinking)

Exercise planning

Relaxation techniques

Scheduling pleasant activities

Improving sleep

Visualizing goals and cultivating healthy optimism

Enhancing your social network

Stress self-assessment tool

Relaxed breathing

Changing negative thinking and cognitive distortions

Increasing intimacy and relationship satisfaction

Increasing sexual satisfaction

Sustaining positive adaptation

the CBT intervention focused on specific misconceptions that have been known to cause distress in cardiac and ICD patients (Conti \& Sears, 2001) and were informed by our clinical work with ICD patients. Ultimately, the goal was to increase self-efficacy and decrease helplessness related to ICD shocks and to the less controllable aspects of their illness.

Counselors were doctoral students, three in clinical psychology and one in exercise sciences, who were trained and supervised by the first author. All had previous psychological counseling experience including clinical practica or previous supervision with the first author when conducting telephone counseling for promoting exercise adherence in vascular patients.

Supervision sessions were typically scheduled biweekly unless therapy sessions with clients were tapering off to monthly intervals, in which case, supervision sessions followed the schedule of the therapy sessions. A therapist manual assisted counselors and specifically addressed: (a) an overview of the heart and specific cardiac abnormalities, (b) the function of the ICD, (c) guidelines for counseling cardiac patients and for assisting patients recovering from an ICD implant, (d) the cognitive model of anxiety and anxiety management interventions, (e) CBT interventions, (f) mindfulness-based stress reduction, (g) interventions to assist people enhance their social supports, (h) therapist's role as active listener, and (i) reading resources.

\section{Measures}

All independent and dependent measures were completed at baseline and 6- and 12-months follow-up. Psychological outcome included measures of anxiety, depression, and PTSD symptoms. Quality of life (QL) was measured by a generic health-related measure.

Anxiety and Depression. Anxiety and depression symptoms were assessed by the 14-item Hospital Anxiety and Depression Scale (HADS; Snaith \& Zigmond, 1994) that was developed for 
use in a non-psychiatric hospital setting. The HADS has good reliability and validity (Bjelland, Dahl, Hang, \& Neckelmann, 2002; Snaith \& Zigmond, 1994). The subscales have also been shown to be sensitive to psychological effects of receiving ICD shocks (Herrmann et al., 1997), and predictive of arrhythmia and shock occurrences (Herrmann et al., 1999). The scales are scored by summing across items with scores greater than 7 representing clinically elevated thresholds (Snaith \& Zigmond, 1994).

Phobic Anxiety. Phobic anxiety was assessed using the eight-item phobic anxiety scale of the Crown-Crisp Experiential Index (CCEI; Crown \& Crisp, 1956). It has well established validity (Burgess, Mazzocco, \& Campbell, 1987; Crown \& Crisp, 1956) and reliability (Alderman, Mackay, Lucas, Spry, \& Bell, 1983; Birtchnell, Evans, \& Kennard, 1988). It has been shown to predict risk of fatal coronary events (Albert, Chae, Rexrode, Manson, \& Kawachi, 2005; Haines, Cooper, \& Meade, 2001). A mean item score is computed and a threshold score of 4 has been associated with a 1.52 times greater risk of SCD (Albert et al., 2005).

Post-traumatic Stress Disorder Symptoms. Post-traumatic stress symptoms were measured using the 22-item Impact of Events Scale-Revised (IES-R) consisting of three sub-scales: avoidance symptoms, intrusiveness symptoms, and hyperarousal symptoms (Weiss \& Marmar, 1997). Scores are computed as the mean item score per scale. A threshold of 1.5 was found to provide the best diagnostic accuracy of PTSD in Vietnam veterans seeking treatment (Creamer, Bell, \& Failla, 2003). The IES-R demonstrates good reliability (Marmar, Weiss, Metzler, Ronfeldt, \& Foreman, 1996) and validity (Creamer et al., 2003). High scores have been associated with an increased risk of mortality in ICD patients (Ladwig et al., 2008). The administration instructions were altered slightly in this study to standardize the nature of the stressor event. Participants were asked to complete the questionnaire "with respect to your arrhythmia (i.e., heart rhythm problem) or its treatment (i.e., having an ICD)."

Quality of Life. QL was assessed by the SF-36 Health Survey version 2 (Ware, Kosinski, \& Dewrey, 2000), a well-validated measure that has extensive normative data. The SF-36 has been found to worsen in ICD patients following ICD shocks (The Antiarrhythmics versus Implantable Defibrillators (AVID) Investigators, 1997). Two summary scores are computed from the items: Physical Component Summary (PCS) score and Mental Component Summary (MCS) score.

Participant Treatment Evaluation and Engagement Measures. A 22 -item treatment evaluation measure was developed specifically for the clinical trial and administered as part of the 12-month outcome assessment battery to participants in the CBT condition. Items asked about the helpfulness and importance of various components of the intervention as well as about topics of discussion during counseling (15 items; treatment evaluation items). Items also asked about strategies the respondents used to help cope with their ICD or cardiac condition (six items; intervention utilization items). There was also an item asking respondents to rate their opinion of the amount of information provided in the ICD psycho-educational booklet. The intervention utilization items were used as an index of treatment engagement. Items were presented in a yes/no response format for the treatment engagement items or a Likert type rating scale for the treatment evaluation items. For analyses, the Likert scaled items were dichotomized as "not at all to somewhat" vs. "very to extremely." The item asking respondents to rate the amount of information included in the ICD psycho-educational booklet was categorized into three levels: "not enough information," "just the right amount of information," or "too much information."

\section{Statistical Analyses}

Data screening revealed positively skewed distributions for the questionnaire scales except for SF-36 PCS, which was normally distributed. A square root transformation was used to normalize the distributions. An intention-to-treat analytic approach was used for the ANOVA analyses. Missing values across follow-up were imputed with expectation maximization, a 
two-step, iterative approach that avoids over-fitting the data and provides realistic and reliable parameter estimates in samples of 100 subjects or more (Tabachnick \& Fidell, 2007). Missing values were not imputed for participants who died over follow-up since the cause of death was assumed to be non-random and potentially related to the phenomenon under study (Schafer \& Graham, 2002).

The associations between gender, treatment engagement, and session attendance rates were tested by either the chi square test of independence or an independent sample $t$-test. The relationship between treatment engagement items and treatment outcome were tested by a series of ANCOVA models with repeated measures whereby the baseline value for the dependent variable was used as the covariate, the treatment engagement item was used as the between subject factor, and the dependent measures at the 6- and 12-month follow-up points were the within subject factors.

The association between number of counseling sessions attended and outcome was tested by a series of hierarchical multiple regression models. The baseline measure of the dependent variable was entered at the first step, number of counseling sessions attended was entered at the second step, and the dependent variable was the 12-month follow-up measure.

The analytic plan of the CBT treatment effect has been reported elsewhere (Irvine, Firestone, Ong, Cribbie, Dorian, et al., 2009). With these sets of analyses, a hypothesis-generating exploratory approach was undertaken in order to help identify potential moderators of treatment outcome (King, Ahn, Atienza, \& Kraemer, 2008). As such no adjustment was made to the alpha level. All data were analyzed using SPSS version 16.0 (SPSS Inc., Chicago, IL).

\section{RESULTS}

\section{Patient Accrual}

Of 680 patients receiving an ICD implant screened, 292 met the study eligibility criteria, 193 consented, and 96 were randomized to the CBT condition. Over follow-up, eight participants died, two in the CBT condition and six in the UCC condition, $\chi^{2}(1, n=193)=2.04, p=.153$. The attrition rate did not differ between CBT $(19.8 \%)$ and UCC $(14.4 \%)$ conditions, $\chi^{2}(1, n=193)$ $=0.98, p=.323$.

The baseline demographic and cardiac variables are displayed in Table 2 for participants in the CBT condition. The only variable that differed between participants who died over the trial versus those who survived was that the former were more likely to be on mood medications $\left(57.1 \%\right.$ vs. $10.3 \%$, respectively), $\chi^{2}(1, n=193)=10.38, p<.001$. Excluding those who died revealed that participants who did not complete the study were less likely than those who completed the study to be married $(45.8 \%$ vs. $79.4 \%), \chi^{2}(2, n=184)=23.84, p<.001$ and noncompleters were found to be younger $(M=56.95, S D=16.71$ vs. $M=62.26, S D=13.61), F(1$, $183)=4.23, p=.042$.

\section{Participants' Evaluations of the CBT Treatment}

Participant responses to the treatment evaluation questions are shown in Table 3. Item endorsement ranged from 66 to 77 respondents. As shown in Table 3, the majority of participants rated both counseling and the psycho-educational booklet as "very to extremely helpful." The topics discussed in counseling by most participants included health information related to the ICD and returning to normal daily activities followed closely by family support issues, stress reduction strategies, and fears related to the ICD. The amount of information covered in the psychoeducational booklet was rated by 73 participants as "just right” by $77.9 \%$, "not enough" by $10.3 \%$, and "too much" by $11.8 \%$. 
TABLE 2. Baseline Demographic and Clinical Characteristics

Variable Cognitive Behavior Therapy Condition $(n=96)$

Mean age $(S D)$

$65.6(14.3)$

Characteristics $\%$

Male

80.0

Employed full or part-time

Greater than high school education

Married

Living alone

NYHA class I

25.8

NYHA class II

40.2

NYHA class III

NYHA unknown

LVEF $^{a}$ Grade 1

LVEF Grade 2 or 3

LVEF Grade 4

LVEF Grade unknown

Primary arrhythmia

VF or cardiac arrest

Sustained VT

Syncope with inducible VT or VF

Syncope with non-inducible VT or VF

Other (e.g., non-sustained VT)

Previous CABG

Previous PCTA/stent

Receiving antidepressant medication

Receiving anxiolytic medication

Currently in cardiac rehab

ICD device therapy over follow-up

An ICD shock or ATP

An ICD shock storm ${ }^{\mathrm{b}}$

6.4

Note. ATP = anti-tachycardia pacing; NYHA = New York Heart Association; PTCA = percutaneous transluminal coronary angioplasty; VF = ventricular fibrillation; VT = ventricular tachycardia. ${ }^{a} \mathrm{LVEF}$ Grade $=$ Left ventricular ejection fraction grade derived from radionuclide ventriculography (RV) or semi-quantitative left ventricular systolic function grade measured by echocardiographic examination. Left ventricular ejection fraction or Grade are measures of the strength of cardiac contraction. Normal ejection fraction is $>50 \%$, normal grade is "grade1"; LVEF < 35\% or LV grade 3 or 4 signify abnormal cardiac function and are associated with a significantly higher risk of cardiac mortality than normal cardiac function.

bShock storm defined as 3 or more ICD shocks within a 24-hour time period. 
Table 3. Participants’ Ratings of the Treatment Evaluation Questionnaire

\begin{tabular}{|c|c|c|}
\hline \multirow[b]{2}{*}{ Treatment Evaluation Items } & \multicolumn{2}{|c|}{$\%$} \\
\hline & $\begin{array}{l}\text { "Not at All to } \\
\text { Somewhat" }\end{array}$ & $\begin{array}{l}\text { "Very to } \\
\text { Extremely" }\end{array}$ \\
\hline $\begin{array}{l}\text { Talking with the counselor about ICD concerns \& fears } \\
\text { was helpful to recovery }(n=77)\end{array}$ & 42.7 & 57.3 \\
\hline $\begin{array}{l}\text { It was helpful for dealing with ICD concerns and fears to } \\
\text { talk about my feelings with the counselor }(n=77)\end{array}$ & 38.6 & 61.4 \\
\hline Information in booklet was helpful to recovery $(n=70)$ & 20.0 & 80.0 \\
\hline \multicolumn{3}{|l|}{ Importance of each to your recovery: } \\
\hline Health/ICD information $(n=72)$ & 20.0 & 80.0 \\
\hline Counseling sessions $(n=72)$ & 41.8 & 58.2 \\
\hline Social support $(n=68)$ & 42.4 & 57.6 \\
\hline Stress reduction $(n=69)$ & 35.8 & 64.2 \\
\hline \multirow[t]{2}{*}{ Relaxation tape helpful to your recovery $(n=66)$} & 67.7 & 32.3 \\
\hline & \multicolumn{2}{|c|}{ Percent "Yes" } \\
\hline \multicolumn{3}{|l|}{$\begin{array}{l}\text { Discussed the following topics with your counselor } \\
\qquad(n=77) \text { : }\end{array}$} \\
\hline Health/Medical information regarding ICD & \multicolumn{2}{|c|}{80.5} \\
\hline Returning back to normal daily activities & \multicolumn{2}{|c|}{74.0} \\
\hline Concerns about living with the ICD & \multicolumn{2}{|c|}{62.3} \\
\hline Stress and coping & \multicolumn{2}{|c|}{51.9} \\
\hline Fears related to your ICD & \multicolumn{2}{|c|}{54.5} \\
\hline Family support & \multicolumn{2}{|c|}{44.2} \\
\hline Other & \multicolumn{2}{|c|}{20.8} \\
\hline \multicolumn{3}{|l|}{ Treatment engagement items } \\
\hline \multicolumn{3}{|l|}{ Used these methods to help me cope with ICD $(n=77)$ : } \\
\hline Correction of faulty thinking & \multicolumn{2}{|c|}{39.0} \\
\hline Progressive muscle relaxation & \multicolumn{2}{|c|}{33.8} \\
\hline Energy boosting exercises & \multicolumn{2}{|c|}{39.0} \\
\hline Imagery guidance/mindfulness & \multicolumn{2}{|c|}{16.9} \\
\hline Record forms & \multicolumn{2}{|c|}{10.4} \\
\hline Modifying cognitive distortions & \multicolumn{2}{|c|}{10.4} \\
\hline
\end{tabular}

\section{Participants Engagement With Treatment}

A frequency count of the number of counseling sessions attended revealed that $29.7 \%$ of participants attended four or fewer counseling sessions, $25.9 \%$ attended five to seven sessions, and $44.4 \%$ attended eight or more sessions. The mean number of therapy sessions attended was 6.24 $(S D=2.17)$. Forty percent attended one face-to-face session. Thus, the majority of participants had solely telephone counseling sessions. 
With respect to reported use of specific intervention strategies, the most frequently endorsed strategies were "correction of faulty thinking" and "energy boosting exercise." As seen in Table 3 , nearly $40 \%$ of participants endorsed using these strategies. The energy boosting strategies included physical exercise, progressive muscle relaxation, scheduling pleasant activities, and restorative sleep facilitating strategies.

\section{Relationship Between Gender and Engagement With Intervention Elements}

Gender was associated with only one of the treatment engagement items. Women were more likely than men to endorse using energy boosting exercises ( $73.3 \%$ vs. $30.6 \%$, respectively), $\chi^{2}(1$, $n=77)=9.25, p=.012$.

\section{Improvement in Psychological Functioning and QL Over Time}

Psychological and QL variables at baseline and 6- and 12-month follow-up within the CBT condition are displayed in Table 4. Improvement over time was observed for all but one of the variables. The only variable that did not change significantly over time was HADS-anxiety.

\section{Differences Between Men and Women in Outcomes}

There were gender by time interaction effects for HADS-depression, $F(1.89,171.70)=5.35, p=$ .006 , PTSD-total symptoms, $F(1.86,173.88)=11.13, p<.001$, PTSD-avoidance, $F(2.00,183.93)=$ $8.72, p<.001$, PTSD-intrusiveness, $F(1.83,168.64)=10.21, p<.001$, PTSD-hyperarousal, $F(1.65$, $152.31)=5.72, p=.048$, and SF-36 MCS, $F(1.79,165.05)=7.38, p<.001$. Women demonstrated greater improvement on these variables. The tests of simple effects analyses using the Sidak test are summarized in Table 4.

\section{Examination of Differences Among Therapists in Treatment Outcome}

None of the ANCOVA analyses testing for differences in the magnitude of improvement in psychological functioning over time among the four therapists were statistically significant.

\section{Relationship Between Treatment Engagement and Treatment Outcome}

The ANCOVA models assessing associations between endorsing using the six treatment strategies and outcome revealed only two significant associations: modifying cognitive distortions was significantly associated with a larger improvement in PTSD total symptoms, $F(1,74)=9.40, p=$ .003 , PTSD-avoidance symptoms, $F(1,74)=6.02, p=.016$, and PTSD intrusiveness symptoms, $F(1,74)=10.15, p=.002$. These associations are tempered by the fact that only eight participants endorsed using the modifying cognitive distortions strategy. The other significant finding was that the use of correcting faulty thinking was associated with significantly greater improvement in PTSD avoidance symptoms, $F(1,74)=4.05, p=.048$. Thirty of the 77 participants endorsed using this strategy to cope with their ICD and cardiac condition over follow-up. Neither number of counseling sessions attended nor attending one face-to-face counseling session was associated with greater improvement in the psychological or QL outcomes.

\section{Summary of Results}

Participants' treatment evaluation ratings of the CBT intervention indicated that many elements of the intervention were judged to be very or extremely helpful and important to their recovery. The majority attended at least five counseling sessions. The only difference in treatment engagement between men and women was that more women reported utilizing energy-boosting 


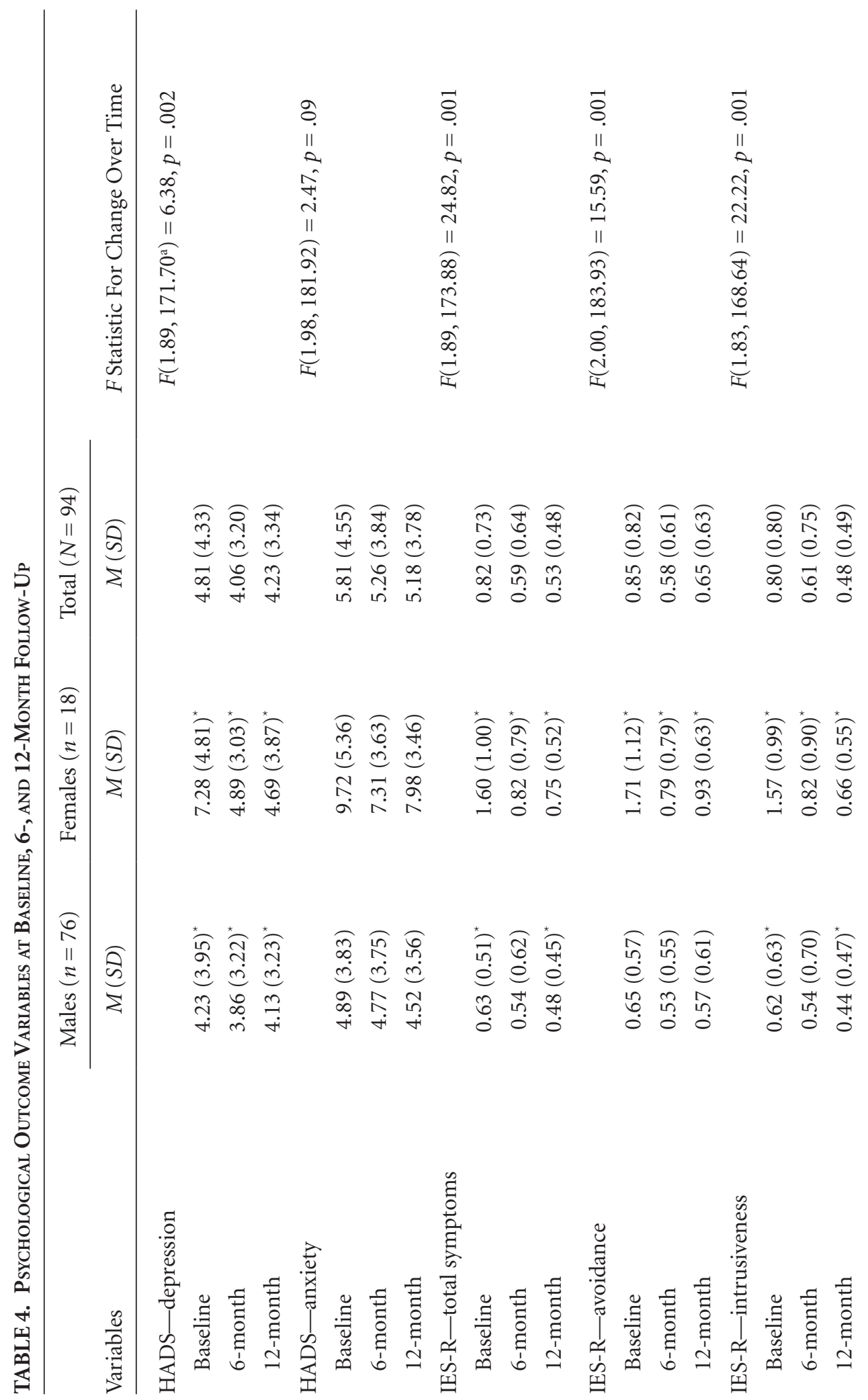




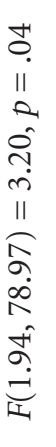

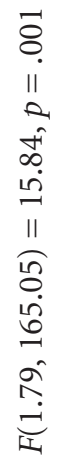

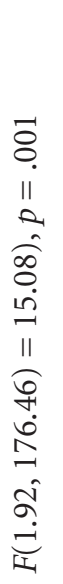

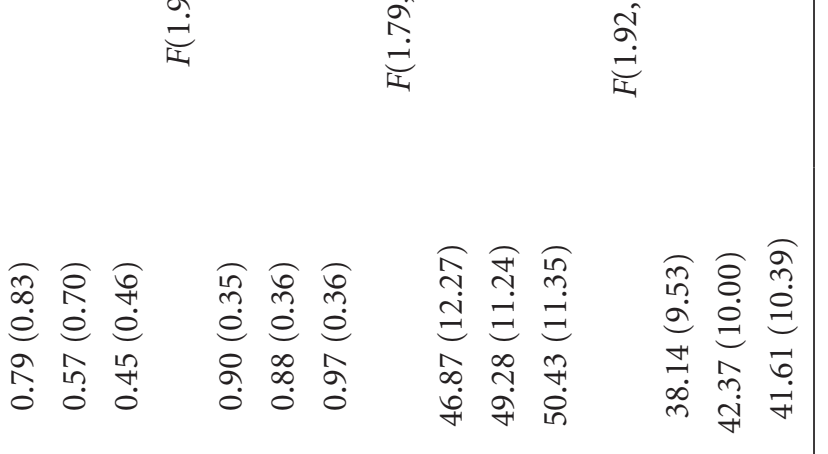

ம்

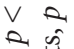

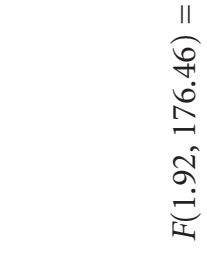

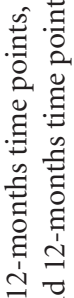

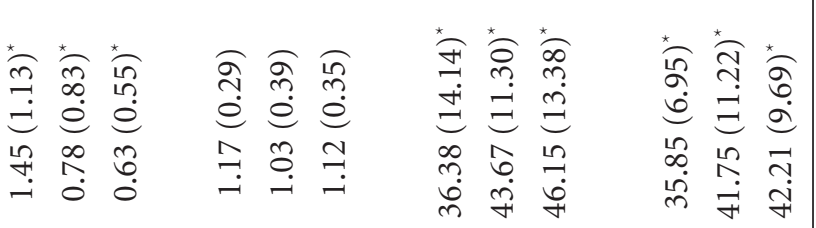

¿.

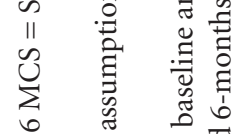

ก)

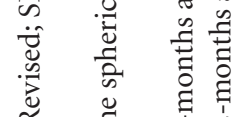

范

$\stackrel{\Xi}{ \pm} \quad$ b

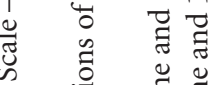

节言

$5)$

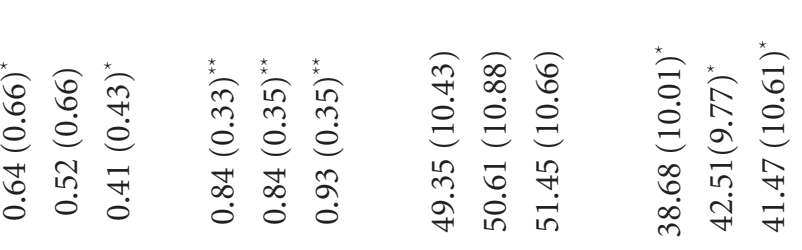

营

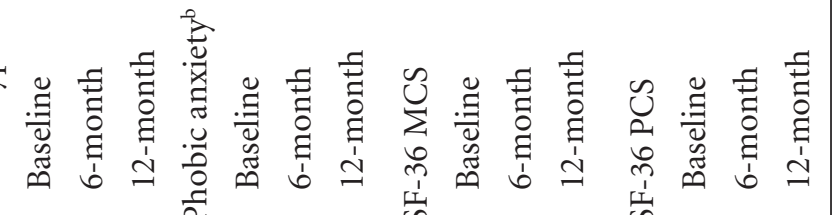

ப

-

II $\quad$ है चี

ن

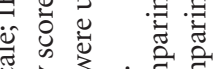

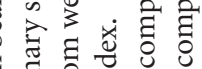

.0

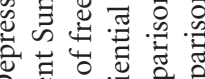

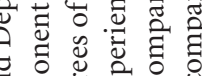

하응

चิ प्य

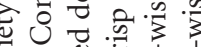

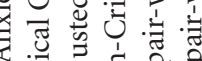

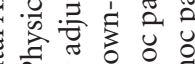

चٓ

की

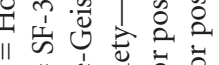

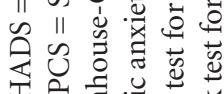

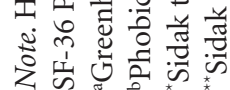


exercises. Utilization of two cognitive strategies was associated with greater improvements in PTSD stress symptoms.

\section{DiscUSSION}

\section{Treatment Acceptance Findings}

Our RCT of a CBT intervention to assist cardiac patients' adaptation to an ICD (Irvine, Firestone, Ong, Cribbie, Johnson, et al., 2009) provided the opportunity to evaluate the acceptability of the intervention and to explore associations between gender and treatment engagement. While approximate indicators of treatment acceptance, our trial participation rate of $66 \%$ and retention rate of $82 \%$ in the CBT condition are high compared to most psychological intervention studies with ICD patients (Pedersen et al., 2007). The CBT intervention delivery via reading material and telephone counseling likely overcame some of the logistical barriers to participation. The early focus on recovery issues and education about the ICD might also have contributed to participant treatment acceptance. In two similar trials with ICD patients that employed telephone counseling and a rehabilitative orientation, high trial participation rates (over 80\%; Kohn, Petrucci, Baessler, Soto, \& Movsowitz, 2000; Lewin, Coulton, Frizelle, Kaye, \& Cox, 2009) and retention rates (77\%; Lewin et al., 2009) were also achieved. A limitation to trial participation and retention rates as indicators of treatment acceptance, though, is that these rates confound willingness to participate in an RCT with acceptance of psychological treatment. Our study represents the next step of specific inquiry regarding participants' opinions of treatment, advocated for studies in medical populations (Andrykowski \& Manne, 2006).

Participant responses to the treatment evaluation items indicated that the CBT intervention was well received, as almost all items were rated by over half of the participants as "very to extremely helpful." The information in the ICD booklet and the health-related information were rated most highly, suggesting that the health-related material was particularly engaging.

Other components of the CBT intervention might have contributed to its acceptability including its ICD-specific content, the client-centered delivery style, and enabling participants to direct the pace and focus of therapy. These features align with factors known to enhance treatment engagement, such as, facilitating a strong therapeutic alliance (Marmar, Weiss, \& Gaston, 1989), reducing treatment resistance (Westra, 2004), and personalizing tailored materials so as to increase participants' attentiveness, involvement, and memory of the material and perhaps also enhancing self-efficacy and motivation (Burg, Campbell, \& Van Assema, 1999). Treatment choice has been suggested to foster autonomy, decrease attrition, increase adherence, and improve overall effectiveness of the treatment program (Pelletier, Tuson, \& Haddad, 1997). Each of these motivational factors might have contributed to the acceptability of our intervention.

Providing participants with an ICD psycho-educational booklet helped ensure that they had access to intervention content even if they did not choose to engage fully with counseling. CBT delivered via written material has been shown to be effective for a variety of psychological problems including depression in older adults (Scogin, Hamblin, \& Beutler, 1987) and general anxiety disorder (Butler, Cullington, Hibbert, Klimes, \& Gelder, 1987). Thus, the multi-modal formats used in our study might have helped meet individual differences in preferred therapy modes and thereby increased treatment acceptability.

\section{Treatment Engagement Findings}

Treatment engagement was indexed by therapy session attendance rates and participants' endorsements of utilizing specific intervention strategies. Session attendance rates suggested a high level 
of engagement as $70 \%$ of participants attended at least $63 \%$ of the sessions and over $40 \%$ attended $100 \%$. Because previous trials of CBT for ICD patients (Chevalier et al., 2006; Frizelle et al., 2004; Kohn et al., 2000; Lewin et al., 2009) did not report session attendance rates, it is impossible to compare rates across trials. This is an area requiring further study in cardiac samples, especially as participation rates for cardiac rehabilitation programs are notoriously poor (e.g., estimated that less than $20 \%$ of eligible patients participate (Lavie, Thomas, Squires, Allison, \& Milani, 2009).

Participants' endorsement of CBT strategies used suggests that $40 \%$ of participants used at least some strategies over follow-up. Our treatment evaluation measure has not undergone psychometric validation. Nor were items originally designed as treatment engagement measures. Moreover, validity might have been affected by the 12-month retrospective recall. Notwithstanding these limitations, our $40 \%$ utilization rate seems reasonable when compared to the range of rates reported by other studies, 49\%-94\% (Kazantzis, Deane, \& Ronan, 2004).

\section{Treatment Engagement and Treatment Outcome Findings}

Improvement in psychological functioning occurred over follow-up, most evident with PTSD symptoms and depressive symptoms, both of which improved more in women than men. Two CBT intervention strategies were associated with greater improvement. Endorsing the use of modifying cognitive distortions was associated with greater improvement in all but one PTSD symptom domain. Endorsing the use of correcting faulty thinking was associated with greater improvement in PTSD avoidance symptoms. The small number of participants who endorsed using modifying cognitive distortions $(n=8)$ and the fact that correcting faulty thinking was significantly associated with only one of the PTSD symptom domains underscores the need to interpret this finding with caution. Notwithstanding this caveat, the results indicate congruence with other treatment studies that have found higher treatment engagement to be associated with better outcomes (Cowan et al., 2008; Meyer et al., 2002; Westra et al., 2007). In other words, participants in our study who remembered these two very specific terms well enough to endorse them at the time of the 12-month follow-up assessment might have been particularly engaged with the intervention. As such, it might not be surprising that these two strategies were associated with greater improvement in PTSD symptoms.

The lack of association between attending a face-to-face counseling session or number of counseling sessions attended and outcome is inconsistent with other studies finding associations between session attendance and better outcome (Alter, Oh, \& Chong, 2009; Witt et al., 2004). The lack of association in our study might be due to factors other than strength of treatment engagement. For example, attending a face-to-face session might have been influenced more by logistical barriers than patient motivational factors. Session attendance rates may reflect a stronger need for counseling than engagement. For instance, early gains in counseling are associated with better outcomes (Kelly, Roberts, \& Ciesla, 2005). Thus, participants who dropped out of counseling earlier might have done so because they did not feel the need for additional counseling sessions. Alternatively, early treatment gains might have confounded the test of the association between session attendance and treatment outcome. The majority of participants attended at least five counseling sessions and so it is also possible that the minimum dose of counseling was sufficient to achieve the full benefit. Anxiety management training has been taught successfully in as few as 3 (Butler et al., 1987) to as many as 8 (Lidren et al., 1994) to 12 sessions (Clark et al., 1994).

\section{Gender Differences in Treatment Engagement and Outcome}

We found no evidence of poorer outcomes or less treatment engagement in women. Quite the opposite, women in our study demonstrated a greater treatment response even after adjusting for their higher baseline scores. Also, the only gender difference on a treatment engagement item was that women were more likely to use energy boosting interventions than men, which could suggest 
stronger engagement by women. Ours is not the first trial to actually find a stronger treatment response in women with cardiovascular disease (Josephson, Casey, Waechter, Rosneck, \& Hughes, 2006). These findings taken together with the studies reporting difficulty recruiting women into treatment programs (Grace et al., 2002; Mohr et al., 2006) might suggest that women are harder to initially engage in lifestyle change programs, but once enlisted, they engage better than men. It is also possible that the adverse psychological treatment outcomes observed for female cardiac patients only apply to specific subgroups of women. For instance, in the Montreal Heart Attack Readjustment Trial (Frasure-Smith et al., 2002), the poorer survival outcomes in women ralative to men were only observed in the high anxious subgroup. Further studies are needed to determine if specific subgroups of women are less likely to benefit from psychological interventions than men.

\section{Limitations of the Study}

This study was conceived after designing our RCT. The variables selected for study were limited to those available. The analytic approach was exploratory and so no adjustments were made to correct for the multiple associations tested. As such, some associations might be spurious and should be considered simply as "sign-posts" to indicate that further scientific attention might be warranted (King et al., 2008).

Another limitation is the lack of a good measure of treatment engagement. We did not have a specific measure of adherence to treatment interventions. While there is no universally agreed upon measure of adherence to psychotherapy interventions, reliable measures that include both clients' reports and therapists ratings are being evaluated (Kazantzis et al., 2004). Researchers need to apply these measures to studies of psychological interventions with cardiac patients.

Finally, as this study was a clinical trial, it is important to acknowledge that patients willing to participate in a trial are not necessarily representative of the general ICD patient population. Thus, the findings of good CBT therapy acceptance could be influenced by the nature of participants willing to participate in a clinical trial. A related additional limitation is that not all participants completed follow-up ( $13 \%$ of those alive at the time of follow-up did not return the follow-up questionnaire), restricting treatment evaluation to those who completed the 12-month follow-up questionnaire.

\section{Conclusion}

A CBT intervention tailored to aid adaptation in recipients of an ICD was accepted well by participants and easily implemented via telephone and media material. Participants appeared to engage well with CBT as evidenced by a high therapy session attendance rate. There was some indication that higher engagement was associated with better outcomes. Multi-modal intervention formats and a choice of intervention strategies might best meet the diverse needs and interests of cardiac patients.

\section{REFERENCES}

Albert, C. M., Chae, C. U., Rexrode, K. M., Manson, J. E., \& Kawachi, I. (2005). Phobic anxiety and risk of coronary heart disease and sudden cardiac death among women. Circulation, 111, 480-487.

Alderman, K. J., Mackay, C. J., Lucas, E. G., Spry, W. B., \& Bell, B. (1983). Factor analysis and reliability studies of the Crown-Crisp Experiential Index (CCEI). British Journal of Medical Psychology, 56, 329-345.

Alter, D. A., Oh, P. I., \& Chong, A. (2009). Relationship between cardiac rehabilitation and survival after acute cardiac hospitalization within a universal health care system. Journal of Cardiovascular Prevention and Rehabilitation, 16, 102-113. 
Andrykowski, M. A., \& Manne, S. L. (2006). Are psychological interventions effective and accepted by cancer patients? Standards and levels of evidence. Annals of Behavioral Medicine, 32, 93-97.

Beck, A. T. (1976). Cognitive therapy and emotional disorders. New York: International University Press.

Berkman, L. F., Blumenthal, J., Burg, M. Carney, R. M., Catellier, D., Cown, M. J., et al. (2003). Effects of treating depression and low perceived social support on clinical events after myocardial infarction: The Enhanced Recovery in Coronary Heart Disease Patients (ENRICHD) Randomized Trial. Journal of the American Medical Association, 289, 3106-3116.

Birtchnell, J., Evans, C., \& Kennard, J. (1988). The total score of the Crown-Crisp Experiential Index: a useful and valid measure of psychoneurotic pathology. British Journal of Medical Psychology, 61, 255-266.

Bjelland, I., Dahl, A. A., Hang, T. T., \& Neckelmann, D. (2002). The validity of the Hospital Anxiety and Depression Scale: An updated literature review. Journal of Psychosomatic Research, 52, 69-77.

Burg, J., Campbell, M., \& Van Assema, P. (1999). The application and impact of computer-generated minimal-personalization nutrition education: A review of the literature. Patient Education and Counseling, $36,145-156$.

Burgess, P. M., Mazzocco, L., \& Campbell, I. M. (1987). Discriminant validity of the Crown-Crisp experimental index. British Journal of Psychiatry, 60, 61-69.

Butler, G., Cullington, A., Hibbert, G., Klimes, I., \& Gelder, M. (1987). Anxiety management for persistent generalised anxiety. British Journal of Psychiatry, 151, 535-542.

Carroll, K. M., Ball, S. A., Martino, S., Nich, C., Babuscio, T. A., Nuro, K., et al. (2008). Computer-assisted delivery of cognitive-behavioral therapy for addiction: A randomized trial of CBT4CBT. American Journal of Psychiatry, 165, 881-888.

Chevalier, P., Cottraux, J., Mollard, E., Brun, S., Burri, H., Restier, L., et al. (2006). Prevention of implantable defibrillator shocks by cognitive behavioral therapy: A pilot trial. American Heart Journal, 151, 191. e1-191.e6.

Clark, D. M., Salkovskis, P. M., Hackmann, A., Middleton, H., Anastasiades, P., \& Gelder, M. (1994). A comparison of cognitive therapy, applied relaxation and imipramine in the treatment of panic disorder. British Journal of Psychiatry, 164, 759-769.

Conti, J. B., \& Sears, S. F. (2001). Understanding and managing the psychological impact of the ICD. Cardiac Electrophysiology Review, 5, 129-133.

Cowan, M. J., Freedland, K. E., Burg, M. M., Saab, P. G., Youngblood, M. E., Cornell, C. E., et al. (2008). Predictors of treatment response for depression and inadequate social support-the ENRICHD randomized clinical trial. Psychotherapy Psychosomatics, 77, 27-37.

Coyne, J. C., \& Lepore, S. J. (2006). Rebuttal: The Black Swan Fallacy in evaluating psychological interventions for distress in cancer patients. Annals of Behavioral Medicine, 32, 115-118.

Creamer, M., Bell, R., \& Failla, S. (2003). Psychometric properties of the Impact of Eevent Scale-Revised. Behavior Research and Therapy, 41, 1489-1496.

Crown, S., \& Crisp, A. H. (1956). A short clinical diagnostic self-rating scale for psychoneurotic patients: the Middlesex Hospital Questionnaire. British Journal of Psychiatry, 112, 917-923.

Cupertino, A. P., Mahnken, J. D., Richter, K., Cox, L. S., Casey, G., Resnicow, K., et al. (2007). Long-term engagement in smoking cessation counseling among rirual smokers. Journal of Health Care for the Poor and Undeserved, 18(4 Suppl), 39-51.

Delsignore, A., Carraro, G., Mathier, F., Znoj, H., \& Schnyder, U. (2008). Perceived responsibility for change as an outcome predictor in cognitive-behavioural group therapy. British Journal of Clinical Psychology, 47, 281-293.

Dunbar, S. B., Jenkins, L. S., Hawthorne, M., Kimble, L. P., Dudley, W. N., Slemmons, M., et al. (1999). Factors associated with outcomes 3 months after implantable cardioverter defibrillator insertion. Heart Lung, 28, 303-315.

Fiorentine, R., Anglin, M. D., Gil-Rivas, V., \& Taylor, E. (1999). Client engagement in drug treatment. Journal of Substance Abuse Treatment, 17, 199-206. 
Frasure-Smith, N., \& Lesperance, F. (2008). Depression and anxiety as predictors of 2-year cardiac events in patients with stable coronary artery disease. Archives General Psychiatry, 65, 62-71.

Frasure-Smith, N., Lesperance, F., Gravel, G., Masson, A., Juneau, M., \& Bourassa, M. (2002). Long-term survival differences among low-anxious, high-anxious and repressive copers enrolled in the Montreal Heart Attack Readjustment Trial. Psychosomatic Medicine, 64, 571-579.

Frasure-Smith, N., Lesperance, F., Prince, R. H., Verrier, P., Garber, R. A., Juneau, M., et al. (1997). Randomised trial of home-based psychosocial nursing intervention for patients recovering from myocardial infarction. Lancet, 350, 473-479.

Frizelle, D. J., Lewin, R. J. P., Kaye, G., Hargreaves, C., Hasney, K., Beaumont, N., et al. (2004). Cognitivebehavioural rehabilitation programme for patients with an implantable cardioverter defibrillator: A pilot study. British Journal of Health Psychology, 9, 381-392.

Gaston, L., Marmar, C. R., Thompson, L. W., \& Gallagher, D. (1988). Relation of patient pretreatment characteristics to the therapeutic alliance in diverse psychotherapies. Journal of Consulting and Clinical Psychology, 56, 483-489.

Grace, S. L., Abbey, S. E., Shnek, Z. M., Irvine, J., Franche, R. L., \& Stewart, D. E. (2002). Cardiac rehabilitation II: referral and participation. General Hospital Psychiatry, 24, 127-134.

Greenberg, R. P., Constantino, M. J., \& Bruce, N. (2006). Are patient expectations still relevant for psychotherapy process and outcome? Clinical Psychology Review, 26, 657-678.

Haines, A. P., Cooper, J., \& Meade, T. W. (2001). Psychological characteristics and fatal ischemic heart disease. Heart, 85, 385-389.

Herrmann, C., Bergmann, G., Drinkmann, A., Dumn, A., Fritzche, K., Kanwische, H., et al. (1999). Anxiety and depression in patients awaiting ICD implantation predict one-year incidence of malignant arrhythmias. Psychosomatic Medicine, 61, 114.

Herrmann, C., von zur Muhen, F., Schaumann, A., Buss, U., Kemper, S., Wantzen, C., et al. (1997). Standardized assessment of psychological well-being and quality-of-life in patients with implanted defibrillators. Pacing and Clinical Electrophysiology, 20, 95-103.

Irvine, J., Basinski, A., Baker, B., Jandciu, S., Paquette, M., Cairns, J., et al. (1999). Psychosocial predictors of sudden cardiac death in CAMIAT. Psychosomatic Medicine, 61, 566-575.

Irvine, J., Dorian, P., Smith, J., O’Brien, B. J., Baker, B., Gent, M., et al. (2002). Quality of life comparison between the implantable cardioverter defibrillator and amiodarone. American Heart Journal, 144, 282-289.

Irvine, J., Firestone, J., Ong, L., Cribbie, R., Dorian, P., Harris, L., et al. (2009). A randomized controlled trial of cognitive behavior therapy tailored to adaptation issues in patients with an implantable cardioverter defibrillator. Poster presented at the American Psychosomatic Medicine 67th Annual Scientific Meeting, Chicago, IL.

Irvine, J., Firestone, J., Ong, L., Cribbie, R., Johnson, S. N., Bilanovic, A., et al. (2009). A randomized controlled trial of cognitive behavior therapy tailored to adaptation issues in patients with an implantable cardioverter defibrillator. Psychosomatic Medicine, 71, A-22.

Irvine, J., Ritvo, P., Sears, S. F., Stanley, J., Dorian, P., \& Newman, D. (2002). An educational booklet for new ICD recipients: A guide for living well with your implantable cardioverter defibrillator (ICD). Unpublished manuscript, Department of Psychology, York University, Toronto, ON, Canada.

Josephson, E. A., Casey, E. C., Waechter, D., Rosneck, J., \& Hughes, J. W. (2006). Gender and depression symptoms in cardiac rehabilitation. Journal of Cardiopulmonary Rehabilitation, 26, 160-163.

Kazantzis, N., Deane, F. P., \& Ronan, K. R. (2004). Assessing compliance with homework assignments: Review and recommendations for clinical practice. Journal of Clinical Psychology, 60, 627-641.

Kelly, M. A. R., Roberts, J. E., \& Ciesla, J. A. (2005). Sudden gains in cognitive behavioral treatment for depression: When do they occur and do they matter? Behaviour Research and Therapy, 43, 703-714.

King, A. C., Ahn, D. F., Atienza, A. A., \& Kraemer, H. (2008). Exploring refinements in targeted behavioral medicine intervention to advance public health. Annals Behavioral Medicine, 35, 251-260. 
Kohn, C. S., Petrucci, R. J., Baessler, C., Soto, D. M., \& Movsowitz, C. (2000). The effect of psychological intervention on patients' long-term adjustment to the ICD: A prospective study. Pacing and Clinical Electrophysiology, 23, 450-456.

Ladwig, K. H., Baumert, J., Marten-Mittag, B., Kolb, C., Zrenner, B., \& Schmitt, C. (2008). Posttraumatic stress symptoms and predicted mortality in patients with implantable cardioverter-defibrillators. Archives General Psychiatry, 65, 1324-1330.

Lavie, C. J., Thomas, R. J., Squires, R. W., Allison, T. G., \& Milani, R. V. (2009). Exercise training and cardiac rehabilitation in primary and secondary prevention of coronary heart disease. Mayo Clinic Proceedings, $84,373-383$.

Leung, A. W., \& Heimberg, R. G. (1996). Homework compliance, perceptions of control, and outcome of cognitive-behavioral treatment of social phobia. Behavior Research and Therapy, 34, 423-432.

Lewin, R. J., Coulton, S., Frizelle, D., Kaye, G., \& Cox, H. (2009). A brief cognitive behavioural preimplantation and rehabilitation programme for patients receiving an implantable cardioverter-defibrillator improves physical health and reduces psychological morbidity and unplanned readmissions. Heart, 95, 63-69.

Lidren, D. M., Watkins, P. L., Gould, R. A., Clum, G. A., Asterino, M., \& Tulloch, H. L. (1994). A comparison of bibliotherapy and group therapy in the treatment of panic disorder. Journal of Consulting and Clinical Psychology, 62, 865-869.

Linden, W., Stossel, C., \& Maurice, J. (1996). Psychosocial interventions for patients with coronary artery disease: A meta-analysis. Archives of Internal Medicine, 156, 745-752.

Marmar, C. R., Weiss, D. S., \& Gaston, L. (1989). Toward the validation of the California Therapeutic Alliance Rating System. Psychological Assessment, 1, 46-52.

Marmar, C. R., Weiss, D. S., Metzler, T., Ronfeldt, H., \& Foreman, C. (1996). Stress responses of emergency services personnel to the Loma Prieta earthquake Interstate 880 freeway collapse and control traumatic incidents. Journal of Traumatic Stress, 9, 63-85.

Meyer, B., Pilkonis, P. A., Krupnick, J. L., Egan, M. K., Simmens, S. J., \& Sotsky, S. M. (2002). Treatment expectancies, patient alliance, and outcome: Further analyses from the National Institute of Mental Health Treatment of Depression Collaborative Research Program. Journal of Consulting and Clinical Psychology, 70, 1051-1055.

Mohr, D. C., Hart, S. L., Howard, I., Julian, L., Vella, L., Catledge, C., et al. (2006). Barriers to psychotherapy among depressed and nondepressed primary care patients. Annals of Behavioral Medicine, 32, 254-258.

Pedersen, S. S., van den Broek, K., \& Sears, S. F. (2007). Psychological intervention following implantation of an implantable defibrillator: A review and future recommendations. Pacing and Clinical Electrophysiology, 30, 1546-1554.

Pelletier, L. G., Tuson, K. M., \& Haddad, N. K. (1997). Client motivation for therapy scale: A measure of intrinsic motivation, extrinsic motivation, and amotivation for therapy. Journal of Personality Assessment, $68,414-435$.

Pilote, L., Dasgupta, K., Guru, V., Humphries, K., McGrath, J., Norris, C., et al. (2007). A comprehensive review of sex-specific issues related to cardiovascular disease. Canadian Medical Association Journal, 176, S1-S44.

Prochaska, J. O., Velicer, W. F., Rossi, J. S., Goldstein, M. G., Marcus, B. H., Rakowski, W., et al. (1994). Stages of change and decisional balance for 12 problem behaviors. Health Psychology, 13, 39-46.

Salkovskis, P. M., Clark, D. M., \& Gelder, M. G. (1996). Cognition-behaviour links in the persistence of panic. Behavior Research Therapy, 34, 453-458.

Schafer, J., \& Graham, J. (2002). Missing data: Our view of the state of the art. Psychological Methods, 7 , 147-177.

Scogin, F., Hamblin, D., \& Beutler, L. (1987). Bibliotherapy for depressed older adults: A self-help alternative. Gerontology, 27, 383-387. 
Sears, S. F., Todaro, J. F., Lewis, T. S., Sotile, W., \& Conti, J. B. (1999). Examining the psychosocial impact of implantable cardioverter defibrillators: A literature review. Clinical Cardiology, 22, 481-489.

Sears, S. F., Vazquez, L. D., Matchett, M., \& Pitzalis, M. (2008). State-of-the-art: Anxiety management in patients with implantable cardioverter defibrillators. Stress and Health, 24, 239-248.

Snaith, R. P., \& Zigmond, A. S. (1994). The Hospital Anxiety and Depression Scale with The IrritabilityDepression-Anxiety Scale and The Leeds Situational Anxiety Scale Manual. London: Nfer Nelson Publishing Company Ltd.

Stefanek, M. E., Jacobsen, P. B., \& Christensen, A. J. (2006). The Society of Behavioral Medicine's "Great Debate": An introduction. Annals of Behavioral Medicine, 32, 83-84.

Tabachnick, B. G., \& Fidell, L. S. (2007). Using multivariate statistics (5th ed.). Boston: Allyn and Bacon.

The Antiarrhythmics versus Implantable Defibrillators (AVID) Investigators. (1997). A comparison of antiarrhythmic-drug therapy with implantable defibrillators in patients resuscitated from near-fatal ventricular arrhythmias. New England Journal of Medicine, 337, 1676-1683.

Ware, J. E., Kosinski, M., \& Dewrey, J. E. (2000). How to score version 2 of the SF-36 Health Survey. Lincoln, RI: QualityMetric Incorporated.

Weiss, D. S., \& Marmar, C. R. (1997). The impact of event scale—revised. In J. Wilson \& T. Keane (Eds.), Assessing psychological trauma and PTSD (pp. 399-411). New York: Guilford.

Westra, H. A. (2004). Managing resistance in cognitive behavioural therapy: The application of motivational interviewing in mixed anxiety and depression. Cognitive Behaviour Therapy, 33, 161-175.

Westra, H. A., Dozois, D. J. A., \& Marcus, M. (2007). Expectancy, homework compliance, and initial change in cognitive-behavioral therapy for anxiety. Journal of Consulting and Clinical Psychology, 75, 363-373.

Witt, B. J., Jacobsen, S. J., Weston, S. A., Killian, J. M., Meverden, R. A., Allison, T. G., et al. (2004). Cardiac rehabilitation after myocardial infarction in the community. Journal of the American College of Cardiology, 44, 988-996.

Acknowledgments. This study was funded by a grant-in-aid from the Heart and Stroke Foundation of Ontario. We would like to also acknowledge the therapists for their invaluable work in the study: Nicole BeaulieuPiotrowski, Jill Firestone, Chantal Arpin-Cribbie and Debra Rotondi-Trevisan.

Correspondence regarding this article should be directed to Jane Irvine, PhD, Department of Psychology, York University, Behavioural Sciences Building, 4700 Keele Street, Toronto, Ontario, Canada, M3J 1P3. E-mail: jirvine@yorku.ca 